\title{
Pigmented paravenous retinochoroidal atrophy: a literature review supported by a unique case and insight

\begin{abstract}
Pigmented paravenous retinochoroidal atrophy (PPRCA) is a rare disorder of unknown origin characterised by bone corpuscle pigmentation accumulation along the distribution of the retinal veins. In addition there are peripapillary pigmentary changes as well as areas of chorioretinal atrophy adjacent to the perivenular pigmentary changes. The finding of PPRCA is usually incidental and does not affect vision. The literature regarding this condition is reviewed. Its natural course has been considered controversial. Observation of the initial insult leading to PPRCA has not previously been reported. A case that provides a photographic record over 20 years of the development of this condition from the initial insult, including the gradual development of the characteristic fundus appearance, is described. The initial presentation was with a sudden reduction of vision and gross diffuse macular oedema in one eye, which was rapidly followed by similar involvement of the fellow eye despite treatment. Thus it was possible to examine the patient at the stage of the initial insult, 5 years before the development of the typical and pathognomonic retinal changes of PPRCA. The clinical and electrophysiological findings are discussed, as also is the relevance of this case to the literature reviewed.
\end{abstract}

Key words Atrophy, Macula, Paravenous, Pigmented, Retinochoroidal

Pigmented paravenous retinochoroidal atrophy (PPRCA) is a rare disorder of unknown origin, the natural course of which is poorly

understood. Diagnosis of this disease is made purely upon its characteristic and peculiar clinical appearance. PPRCA was first described ${ }^{1}$ in 1937 in a 47-year-old man who developed alopecia areata and in whom there was the incidental finding of a bilateral and peculiar fundus appearance, the features of which have since become considered typical of this disease, i.e. bone corpuscle pigmentation accumulation along the distribution of the retinal veins, beginning some distance from the optic nerve head. In addition peripapillary pigmentary changes were seen, as well as areas of chorioretinal atrophy adjacent to the perivenular pigmentary changes. This condition was termed retinochoroiditis radiata ${ }^{1}$ and in the light of the patient's positive reaction to tuberculin and family history of tuberculosis, it was suggested that the aetiology might have been a tuberculous periphlebitis. $\mathrm{Chi}^{2}$ also considered the possibility of an inflammatory cause and presented a case with a proposed syphilitic aetiology. Subsequently numerous other titles were assigned to this condition, such as congenital pigmentation of the retina, ${ }^{3}$ in which a congenital anomaly was suggested, melanosis of the retina ${ }^{4}$ and paravenous retinal degeneration, in which a degenerative aetiology was proposed. ${ }^{5}$ This confusing battery of terms has been superseded by the now generally accepted descriptive term pigmented paravenous retinochoroidal atrophy (PPRCA), which was first proposed by Franceschetti. ${ }^{6} \mathrm{He}$ was the first to review the relatively small number of reported cases at the time, and concluded that they represented a similar disease process.

This, however, is not apparent when reviewing the subsequent disparate and more numerous case reports. The authors are aware of 90 cases reported in the literature, in which young men are most commonly affected with only 10 cases occurring in females. ${ }^{3,7-13}$ The finding of PPRCA is usually incidental and does not affect vision. The natural course of this condition has been considered controversial. ${ }^{4,14,15}$ Some authors have suggested that PPRCA has a congenital origin $^{8,16-18}$ and others a primary retinal degeneration. ${ }^{13}$ The condition has been described as both progressive and nonprogressive in both children and the elderly by different authors. ${ }^{13,14,18,19}$ In most cases the
A.T. Murray

G.R. Kirkby

Birmingham and Midland

Eye Hospital

Birmingham, UK

Mr Aidan T. Murray, FRCOphth

Moorfields Eye Hospital

City Road

London EC1V 2PD, UK

e-mail: AidanMurray@

Doctors.org.uk

Received: 23 November 1999 Accepted in revised form: 21 March 2000 


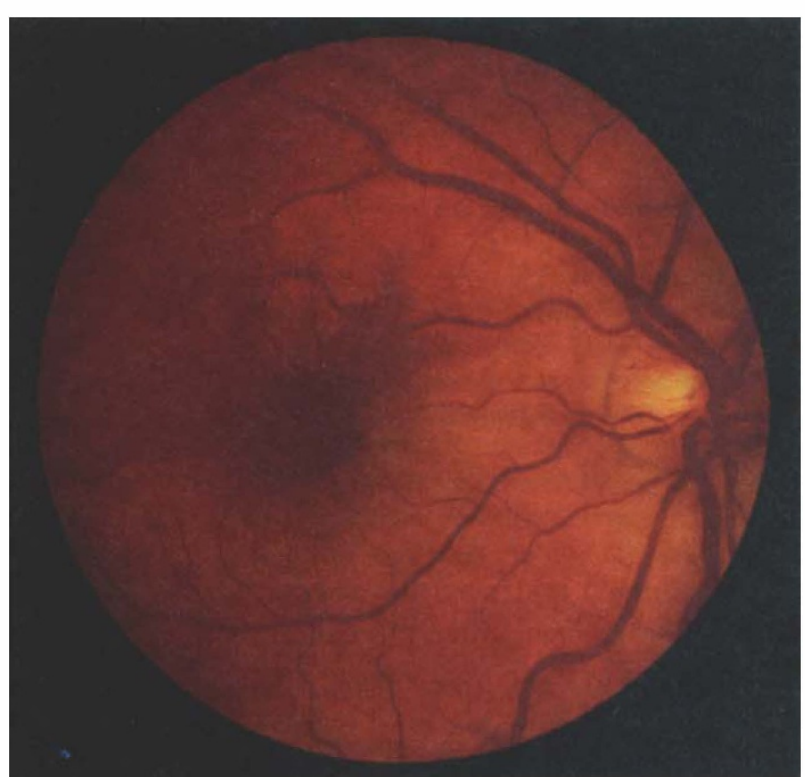

Fig. 1. Fundal appearance of the right eye on admission. The pale ischaemic retina is sparing the fovea.

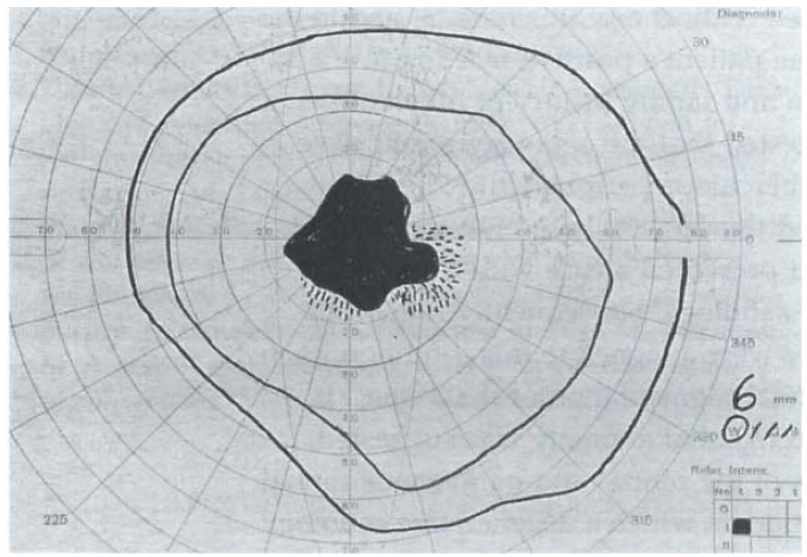

(a)

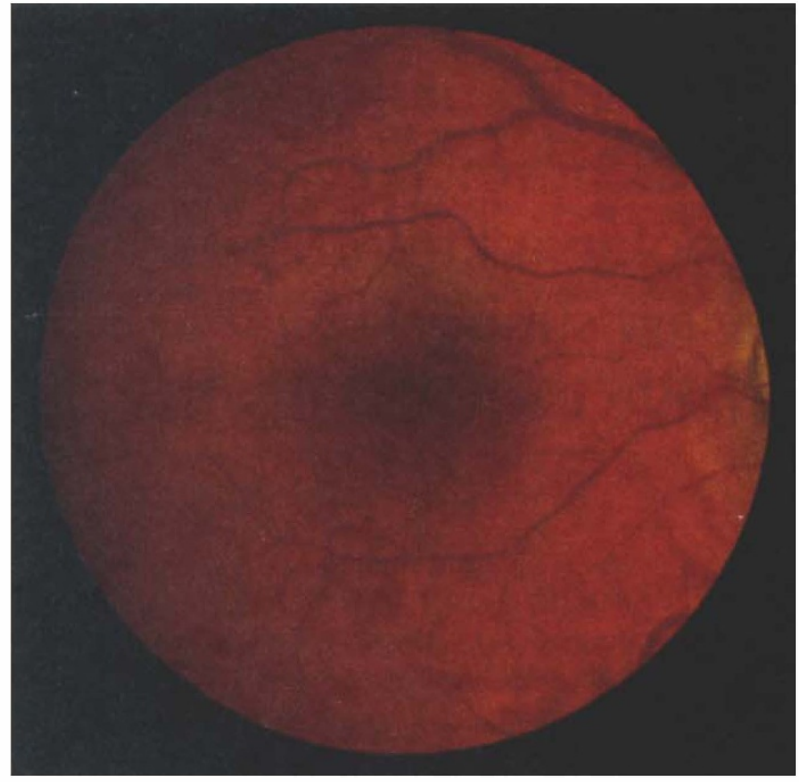

Fig. 2. Two days after admission, the ischaemic retina on the right eye now involves the whole macula.

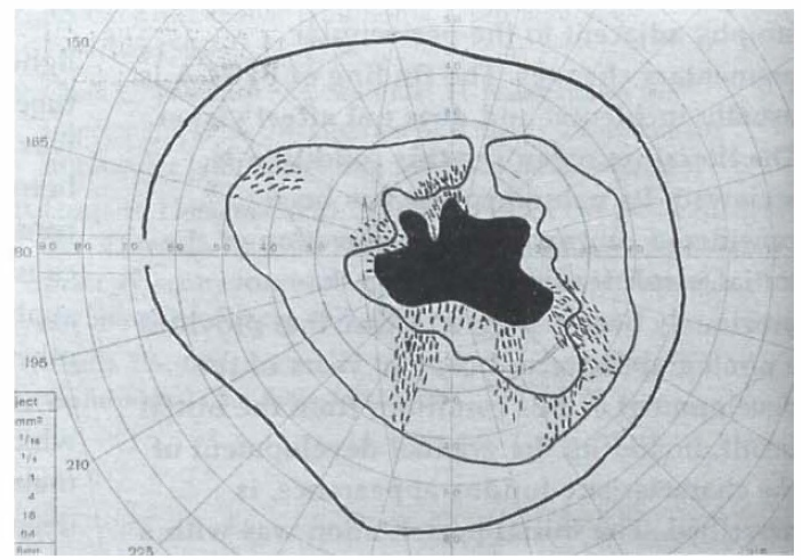

(b)

Fig. 3. (a) Goldmann visual field of the right eye showing a central scotoma with a normal peripheral field. (b) Goldmann visual field of the left eye showing a central scotoma with some extension into the peripheral field.

optic nerve, the unaffected retina and the calibre of the retinal vessels are normal. In only a very small minority of cases has the macula been reported to be affected, ${ }^{7,8,13,16,17,20,21}$ with only 6 cases of macular involvement to our knowledge previously having been reported. In most cases the condition does not progress.

Reports in the literature which include electrodiagnostic data show a spectrum of results ranging from normal to extinguished electroretinograms (ERGs) and both normal and abnormal electrooculograms (EOGs). A review of 10 such cases ${ }^{22}$ considered that this reflected either different degrees of involvement or different stages of development.

There have been 3 reports of the familial occurrence of PPRCA; $;, 8,16$ however, the majority of cases have been sporadic. As a result there has been varied speculation as to the inheritance by dominant, recessive, $X$-linked and $Y$-linked modes of transmission. No convincing evidence for one of these modes of transmission has, however, been made.
The confusing array of cases and proposed aetiologies has led to the suggestion that PPRCA represents several different afflictions, produced by a variety of genetic and acquired disorders, ${ }^{18,23-25}$ all resulting in a similar fundoscopic appearance, and that probably some of these cases represent a localised or geographic form of pigmentary retinal dystrophy or dysgenesis whereas others represent post-inflammatory scarring.

Herein we describe a case that evolved over a 20 year period, which offers a unique insight into the development of this condition.

\section{Case report}

On 18 March 1977 a 17-year-old white Caucasian female presented with a history of a sudden marked reduction in the vision of her left eye, which had occurred 3 days previously. Her vision was $6 / 5$ in the right eye and hand movements in the left. Examination revealed no vitreous cells but diffuse gross oedema of the posterior pole of the 


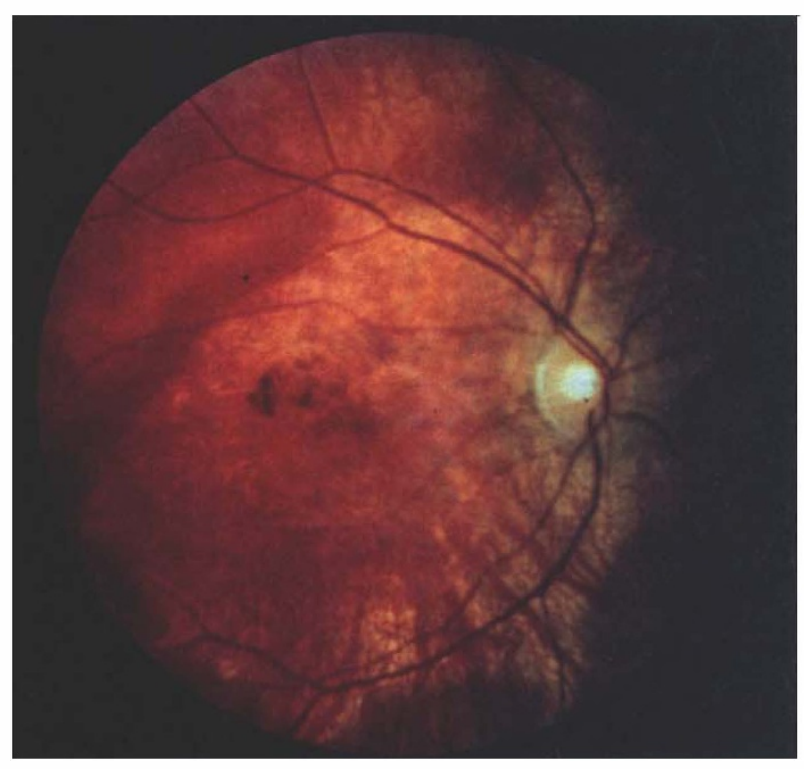

Fig. 4. Right eye 5 years after acute presentation showing parafoveal pigmentation with segmental choroidal atrophy.

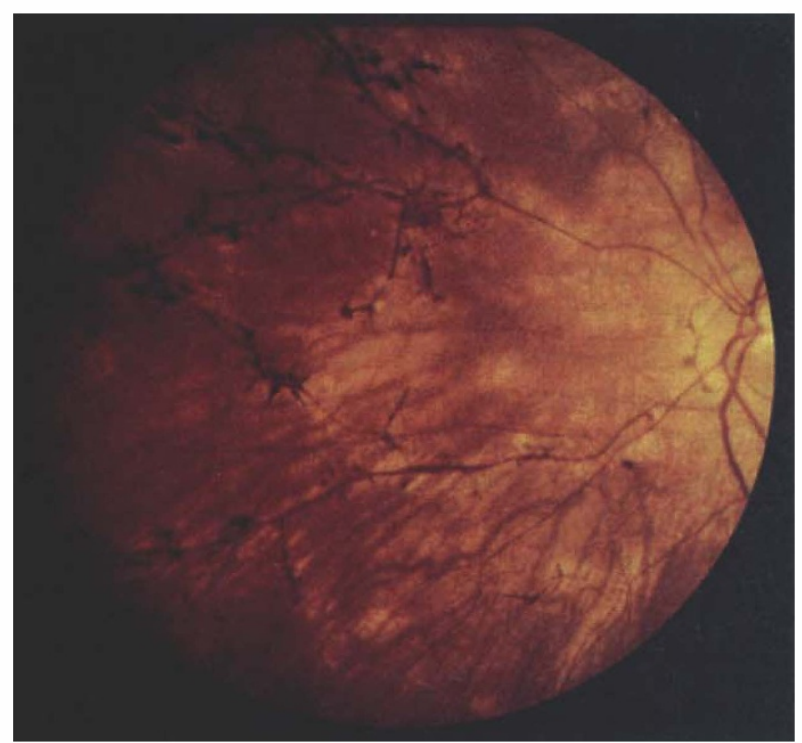

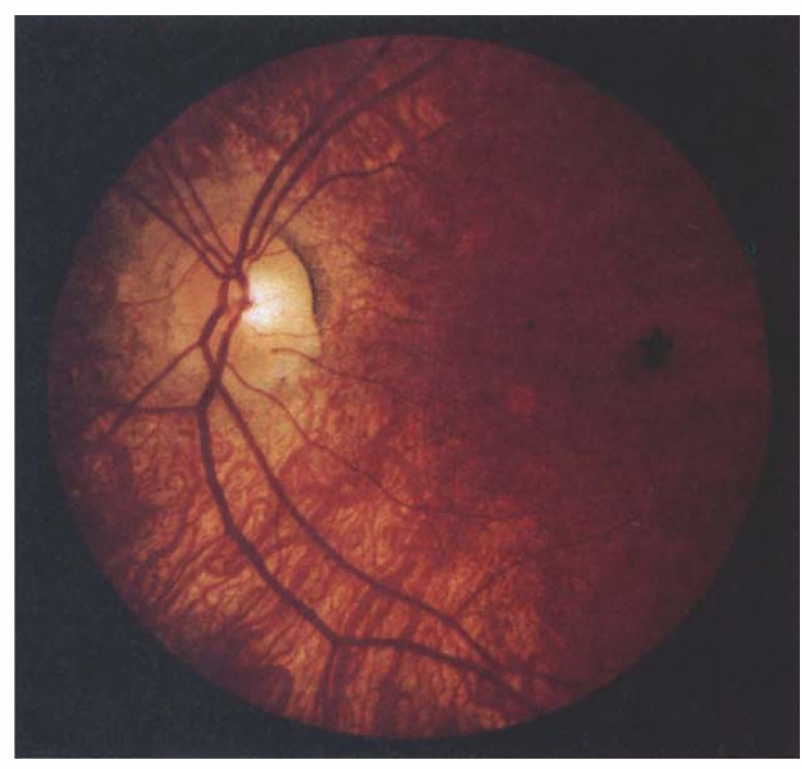

Fig. 5. Left eye 5 years after acute presentation showing parafoveal pigmentation with segmental choroidal atrophy.

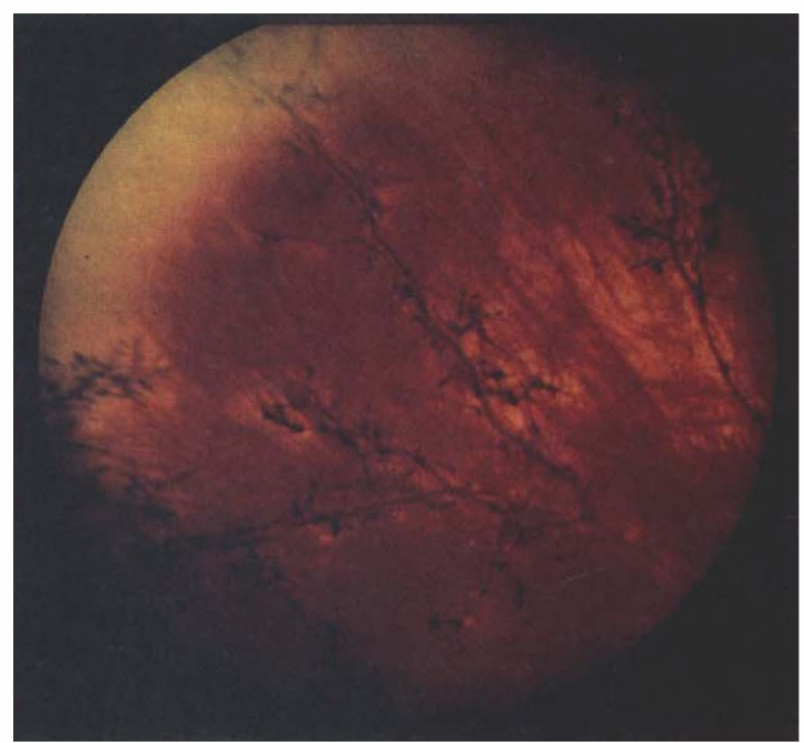

Fig. 6. Left peripheral retina 20 years after acute presentation, showing bone corpuscle pigmentation accumulation along the distribution of the retinal veins with adjacent areas of chorioretinal atrophy.

left eye. No abnormality of the right eye was seen. She had no family history of ophthalmic disease apart from a paternal aunt with primary open angle glaucoma. She had 2 unaffected siblings (male and female) and later she had 2 currently unaffected daughters (now aged 17 and 13 years). She was on no medication and was not taking oral contraception. A provisional diagnosis of a left branch artery occlusion was made.

One week after the initial episode she became aware of a shadow in front of the right eye and attended the casualty department, from which she was admitted. Initially her vision was $6 / 5$ in the right eye and hand movements in the left. The patient felt fit and well. Fundal examination was described as revealing a granular membrane over the posterior pole (Fig. 1). She was admitted and given high-dose systemic prednisolone and vasodilators; however, her vision rapidly deteriorated in her right eye to $6 / 60$ (Fig. 2). The fundal appearances showed a large area of pale ischaemic retina which initially spared the fovea (Fig. 1) and which 2 days later encompassed the whole macula (Fig. 2). Sarcoidosis was actively considered as a possible differential diagnosis but no immunological or haematological evidence was found for this. The chest radiograph was normal and syphilis serology was negative. Urinalysis and blood pressure were normal. The only abnormal finding was a raised IgM level of $2.63 \mathrm{~g} / 1$ (normal range $0.5-1.5 \mathrm{~g} / \mathrm{l}$ ). In addition ANF was negative and there were no Le cells (polymorphs with denatured nuclei seen in systemic lupus erythematosus).

After approximately 3 weeks she was discharged on $5 \mathrm{mg}$ t.d.s. prednisolone o.d. Soon after discharge Goldmann visual fields (Fig. 3) revealed large bilateral central scotomata with the peripheral field being normal 
Table 1. A comparison of the electrodiagnostic results from first diagnosis to most recent results 20 years later

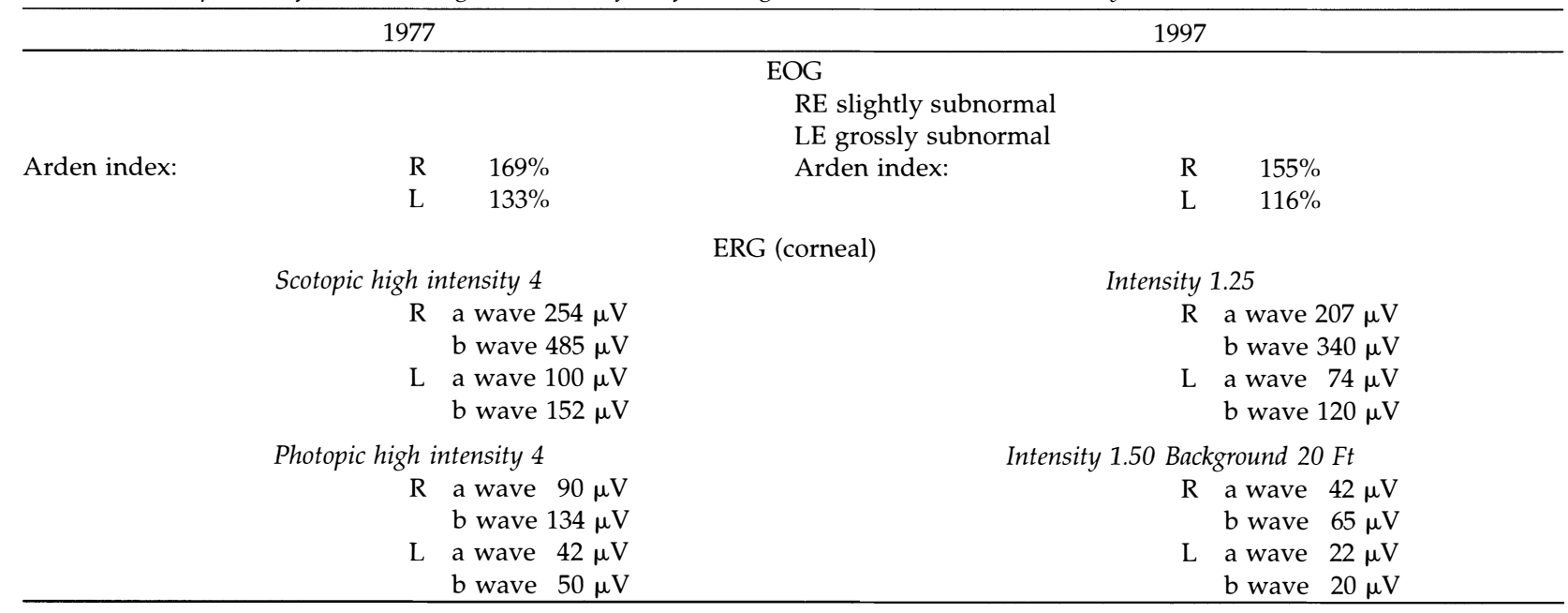

in the right eye but with some peripheral extension of the scotoma into the periphery in the left eye. Her visual acuity was $6 / 60$ in the right eye and 3/60 in the left. Fluorescein angiography was reported as being suggestive of choroidal atrophy and arterial occlusion (the images have unfortunately been lost).

Table 1 summarises the findings of the electrodiagnostic tests performed in 1977 and 1997. There is some difficulty in comparing these results as such tests were in their infancy in 1977; however, there does not appear to be a marked deterioration and the findings show an absent pattern ERG consistent with the bilateral maculopathy. The ERG for the right eye is within normal limits whilst responses from the left eye are grossly reduced and delayed to all stimuli (rod and cone responses equally affected). This shows that in the left eye there is a widespread retinopathy. These findings would be consistent with bilateral ischaemic maculopathy with the ischaemia being more widespread in the left eye. Interestingly the oscillatory potentials are normal in the right eye (although markedly reduced in the left eye), which would imply that vasculitis is unlikely to be an aetiological factor.

At this stage the differential diagnosis was very limited. It was apparent that the patient had suffered a bilateral ischaemic maculopathy, but the aetiology remained elusive, as it does to this day. Four months after the episode the fundal appearance was described as showing 'very fine macula thinning (would pass for normal if seen first)'.

The patient was discharged but reviewed again when she became pregnant in 1982, by which time some parafoveal pigmentation had developed and segmental choroidal atrophy was apparent (Figs. 4,5 ). In addition some peripheral pigment associated with the blood vessels was noted. Her visual acuity had remained stable at 6/60 in the right eye and 3/60 in the left. In 1986 further photographs showed an increase in pigmentation and these changes are further emphasised by the most recent photographs taken in 1997 (Fig. 6). These show the development of the characteristic changes seen in PPRCA. Her visual fields have remained unchanged.
B-scan ultrasonography revealed no elevation of the macular lesions and no optic disc drusen; a single case of the latter association has been reported. ${ }^{24}$

\section{Discussion}

This case provides a unique insight into the development of PPRCA, it being the only reported case in which the full evolution of this condition has been reported. Of particular interest was the absence of any apparent paravascular inflammation at the time of the initial insult and yet, as can be seen from Fig. 6, paravascular retinochoroidal atrophy is one of the most prominent features of the final fundal appearance and of PPRCA as a whole. Although this case provides a fascinating insight into PPRCA it can be seen from the review of the literature that it is not typical. The patient was female (10/90 in the literature $)^{3,7-13}$ and there was macular involvement (6/90 in the literature) $)^{7,8,13,16,17,20,21}$ with an associated poor visual outcome, whereas in the majority of cases vision is normal, with the finding of PPRCA being incidental, and no progression in the condition would be expected.

As previously discussed PPRCA appears to represent several different afflictions, produced by a variety of genetic and acquired disorders, ${ }^{18,23-25}$ all resulting in a similar fundoscopic appearance. It has not been possible to attribute a precise diagnosis to the initial insult in this case. The final appearance was probably the result of post-inflammatory scarring. Whilst this would explain the macular changes, there was little evidence of vascular inflammation in the initial stages of the disease which would lead to the typical PPRCA picture. With regard to the macular insult, one condition which might fit the apparent pigment epithelial swelling seen in Fig. 1, is acute multifocal placoid pigment epitheliopathy (AMPPE). AMPPE has not, however, been described in the literature as leading to PPRCA. The condition did not have a multifocal placoid appearance (although the size, shape and distribution of the lesions seen in AMPEE may be variable), and AMPPE is normally associated with a good visual outcome; a long-term follow-up by Gass ${ }^{26}$ 
showed that less than $4 \%$ of eyes achieved a visual acuity of $<20 / 30$ after 5 years. Most patients with AMPEE also have normal EOGs and ERGs during the acute phase of the disease, although subnormal EOGs have been reported. ${ }^{27}$ The presence in the acute phase of the disease of an isolated raised IGM level would be consistent with a primary immune response. Unfortunately this would be consistent with the acute inflammation that may occur either in PPRCA ${ }^{28,29}$ or in AMPPE. Interestingly, vitreous inflammatory cells, which are found in $50 \%$ of patients with $\mathrm{AMPEE},{ }^{27}$ were not present in this case.

It would appear from the numerous associations of PPRCA that it does not represent a single disease entity, but is rather the end result of a variety of inflammatory or dystrophic processes with their attendant

paravascular pigmentary changes. It is well recognised that pigmentary changes in the fundus can be produced by a number of disparate insults, such as retinitis pigmentosa and trauma-caused pseudo-retinitis pigmentosa. In a similar fashion PPRCA is the final fundal appearance produced by a number of aetiological insults. ${ }^{13}$ It appears likely that there are cases of paravascular inflammation or vasculitis associated with well-known conditions (e.g. sarcoidosis) but for which at the time of diagnosis no markers of the disease are able to be demonstrated. This case was brought to the clinicians' attention at the acute stage of the initial insult (because of macular involvement, etc.) and interestingly there was no clinical evidence of vasculitis. One could speculate that where macular involvement is not present, as occurs in most cases leading to PPRCA, the fundal changes at the time of the initial insult may be macroscopically unremarkable - hence the usual incidental finding of the characteristic clinical appearance of the disease approximately 5-10 years after the initial inflammatory insult.

This case demonstrates that there is progression of the pigment clumping and paravenous retinochoroidal atrophy, which lead to the characteristic pathognomy. However, it would appear that these are gradual subsequent degenerative changes arising from an acute episode, rather than true progression. Although this would explain the progressive fundoscopic changes noted by Pearlman et al. ${ }^{14}$ in one of their cases, it would not adequately explain the progressive visual field constriction they noted. However, a degree of degenerative change would be consistent with the 'progressive' case noted by Noble and Carr. ${ }^{7}$ Certainly the overwhelming findings of the case reports presented in the literature show visual fields to be either normal or showing multiple focal scotomas. ${ }^{14}$ In addition there were no common abnormalities of dark adaptation.

Electrodiagnostic tests are usually normal or only mildly affected in PPRCA, but in some instances have been found to be markedly subnormal. It can be seen from this case report that there has not been definitive change in the electrodiagnostic tests over a 20 year period, the initial tests being performed soon after the initial insult and some 5 years before the development of the pathognomonic retinal changes. Thus it would appear that the changes seen in electrodiagnostic tests in the different cases reported, do indeed simply represent the different degrees of involvement rather than a stagedependent effect.

\section{Conclusion}

PPRCA is a rare condition in which the aetiology remains elusive. This unique case, however, which is the only reported case in which the condition has been seen from the initial insult, does broaden our understanding of the development of PPRCA. It can now be confirmed that it is not exclusively a congenital condition, as has been suggested. ${ }^{3}$ It lends credence to the view that the condition is not progressive as such, but that the initial insult leads to the slow development of the characteristic atrophic changes and pigmentary distribution over a period of approximately 5-10 years. Many case reports have attempted to look for a systemic cause for this condition, citing patients in whom there was a strong family history of tuberculosis, ${ }^{1}$ a previous history of syphilis, ${ }^{2}$ or who developed PPRCA following rubeola retinopathy. ${ }^{30}$ However, from the large number of cases reported, there does not appear to be any known systemic cause for this condition; patients are usually asymptomatic and the finding of PPRCA is incidental. The presence of previous disease, when this is rarely found, appears to be a confounding rather than a causative association.

\section{References}

1. Hewitson-Brown T. Retinochoroiditis radiata. Br J Ophthalmol 1937;21:645.

2. Chi Hsin-Hsing. Retinal choroiditis radiata. Am J Ophthalmol 1948;31:1485.

3. Morgan OG. Congenital pigmentation of the retina. Proc $\mathrm{R}$ Soc Med 1948;41:726.

4. Brognoli C. Sopra un case di pigmentazione anomala del fondo oculare (melanosi della retina). Arch Ofttal 1949;53:99-119.

5. Weve H. Degeneratio retinae paravenosa. Mod Probl Ophthalmol 1957;1:664.

6. Franceschetti A. A curious affection of the fundus oculi. Helicoid peripapillary chorioretinal degeneration. Its relation to pigmentary paravenous chorioretinal degeneration. Doc Ophthalmol 1962;16:81.

7. Noble KG, Carr RE. Pigmented paravenous chorioretinal atrophy. Am J Ophthalmol 1983;96:338-44.

8. Traboulsi EI, Maumenee IH. Hereditary pigmented paravenous chorioretinal atrophy. Arch Ophthalmol 1986;104:1636-40

9. Law F. Discussion of communication of Morgan. Proc R Soc Med (Sec Ophthalmol) 1948;41:727.

10. Almaric P, Schum U. Pigmentiente paravenose Netz-und Aderhautatropie. Klin Monatsbl Augenheilkd 1968;153:770.

11. Parafitta $\mathrm{M}$, et al. Pigmented paravenous retinochoroidal atrophy. Optom Vis Sci 1993;70:75-8.

12. Young WO, Small KW. Pigmented paravenous chorioretinal atrophy (PPRCA) with optic disc drusen. Ophthalmic Paediatr Genet 1993;14:23-7.

13. Lessel LR, Thaler A, Heilig P. ERG and EOG in progressive paravenous retinochoroidal atrophy. Doc Ophthalmol 1986;62:25-9. 
14. Pearlman JT, Heckenlively JR, Bastek JV. Progressive nature of pigmented paravenous retinochoroidal atrophy. Am J Ophthalmol 1978;85:215-7.

15. Pearlman JT, Kamin DF, Kopelow SM, Saxton J. Pigmented paravenous retinochoroidal atrophy. Am J Ophthalmol 1975;80:630-5.

16. Skalka HW. Hereditary pigmented paravenous retinochoroidal atrophy. Am J Ophthalmol 1979;87:286-91.

17. Noble KG. Hereditary pigmented paravenous chorioretinal atrophy. Am J Ophthalmol 1989;108:365-9.

18. Small KW, Anderson WB Jr. Pigmented paravenous retinochoroidal atrophy: discordant expression in monozygotic twins. Arch Ophthalmol 1991;109:1408-10.

19. Gass JDM. Stereoscopic atlas of macula diseases: diagnosis and treatment. St Louis: Mosby, 1997:379.

20. Chen M-S, Yang C-H, Huang J-S. Bilateral macula coloboma and pigmented paravenous retinochoroidal atrophy. $\mathrm{Br} \mathrm{J}$ Ophthalmol 1992;76:250-1.

21. Johansen J, Lund-Anderson C, Autzen T. Pigmented paravenous retinochoroidal atrophy. Acta Ophthalmol 1988;66:474-7.

22. Miller SA, Stevens TS, Myers F, Nieder M. Pigmented paravenous retinochoroidal atrophy. Ann Ophthalmol 1978;10:867.
23. Nucci P, Mannitto MP, Piantanida A, Bracato R. Macula dysplasia and pigmented paravenous retinochoroidal atrophy. Ophthalmol Genet 1994;15:161-4.

24. Young WO, Small KW. Pigmented paravenous retinochoroidal atrophy (PPRCA) with optic disc drusen. Ophthalmic Paediatr Genet 1993;14:23-7.

25. Heckenlively JR, Kokame GT. Pigmented paravenous retinochoroidal atrophy. Doc Ophthalmol Proc Ser 1984;40:235-41.

26. Gass JDM. Acute posterior multifocal placoid pigment epitheliopathy: a long term follow-up study. In: Fine SL, Owens SL, editors. Management of retinal vascular and macular disorders. Baltimore: Williams and Wilkins, 1983.

27. Gass JDM. Stereoscopic atlas of macula diseases: diagnosis and treatment. St Louis: Mosby, 1997:668-75.

28. Limaye SR, Mahmood MA. Retinal microangiography in pigmented paravenous chorioretinal atrophy. Br J Ophthalmol 1987;71:757-61.

29. Yamaguchi K, Hara S, Tanifuji Y, Tamai M. Inflammatory pigmented paravenous retinochoroidal atrophy. $\mathrm{Br} \mathrm{J}$ Ophthalmol 1989;73:463-7.

30. Foxman SG, Heckenlivelly JR, Sinclair SH. Rubeola retinopathy and pigmented paravenous retinochoroidal atrophy. Am J Ophthalmol 1985;99:605-6. 